\title{
Commentary on 'Surgical treatment of primary uterine prolapse: a comparison of vaginal native tissue surgical techniques'
}

\author{
Matthew L. Izett ${ }^{1,2}$ (D) \\ Received: 14 April 2019 / Accepted: 17 April 2019/Published online: 23 May 2019 \\ (C) The International Urogynecological Association 2019
}

In this large national database study, Husby et al. compare various native tissue surgical approaches for the treatment of primary uterine prolapse [1]. Combining registry data with clinical details from a national urogynaecology database, they include vaginal hysterectomy (VH), Manchester-Fothergill procedure (MP) and sacrospinous hysteropexy (SH). With increasing scrutiny of mesh-augmented prolapse procedures, and a desire from women to explore uterine-preserving surgery, large studies of native tissue procedures are needed. A recent systematic review compared hysterectomy with uterine preservation, and found advantages to uterine-preserving techniques [2]. Therefore, evidence such as that found in this informative study, provide important data to support decision making for patients and clinicians when considering surgery for primary uterovaginal prolapse.

The authors identified 7247 cases over a 6-year period (2010 to 2016) from the Danish National Patient Registry. Data were obtained by cross referencing cases to the Danish Urogynaecological Database and collected up to 2017. Only those patients undergoing a primary apical prolapse operation without a concurrent continence procedure were included, and adjustments were made for a range of relevant variables. The primary outcome for this study was reoperation for apical prolapse, although data for reoperation for all compartments were analysed. The median follow-up was 43 months (range 1-90). Reoperation for apical prolapse was far more likely for those undergoing SH compared with MP [hazard ration (HR) 40.2, confidence interval (CI) 21.674.7] and VH (HR 8.5, CI: 6.0-12.1), as well as for prolapse of other compartments. Comparing VH to MP, VH was associated with a higher risk of subsequent apical prolapse

Matthew L. Izett

m.izett@ucl.ac.uk

1 Urogynaecology Research Fellow, University College London Hospital , London, UK

2 University College London, London, UK surgery (HR 4.2, CI: 2.4-7.2), as well as for other compartments. At 5 years, the risk of reoperation for prolapse of any compartment was $32 \%$ for $\mathrm{SH}, 11 \%$ for $\mathrm{VH}$ and $7 \%$ for MP.

The authors of this study conclude that due to high rates of reoperation, the use of $\mathrm{SH}$ should be restricted pending availability of supportive evidence. They also make a case for the superiority of MP over $\mathrm{VH}$ due to lower rates of reoperation. With a large unselected cohort, and data from a national database with high rates of complete data $(>90 \%)$, the evidence presented is compelling. Limitations of this study include a lack of clarity with respect to surgical techniques, particularly regarding apical support at $\mathrm{VH}$; however, the authors suggest this is routine practice on the basis of more detailed studies undertaken in a similar cohort [3]. With the evolving controversies around mesh and a continued desire amongst many women to avoid hysterectomy, further evidence to support this study's findings would be welcome.

\section{References}

1. Husby et al. surgical treatment of primary uterine prolapse: a comparison of vaginal native tissue surgical techniques.

2. Meriwether KV, Antosh DD, Olivera CK, Kim-Fine S, Balk EM, Murphy M, et al. Uterine preservation vs hysterectomy in pelvic organ prolapse surgery: a systematic review with meta-analysis and clinical practice guidelines. Am J Obstet Gynecol. 2018;219(2):12946.

3. Tolstrup CK, Husby KR, Lose G, et al. The Manchester-Fothergill procedure versus 262 vaginal hysterectomy with uterosacral ligament suspension: a matched historical cohort 263 study. Int Urogynecol J. 2018;29:431-40. https://doi.org/10.1007/s00192017-3519-9.

Publisher's note Springer Nature remains neutral with regard to jurisdictional claims in published maps and institutional affiliations. 